\title{
IPHAS A-type Stars with Mid-IR Excesses in Spitzer Surveys
}

\author{
Antonio S. Hales ${ }^{1}$, Michael J. Barlow, Janet E. Drew, \\ Yvonne C. Unruh, Robert Greimel, Michael J. Irwin and \\ Eduardo González-Solares
}

${ }^{1}$ Joint ALMA Observatory, Av. El Golf 40, Piso 18, Santiago, Chile, email: ahales@alma.cl

\begin{abstract}
The Isaac Newton Photometric H-Alpha Survey (IPHAS) provides $\left(\mathrm{r}^{\prime}-\mathrm{H} \alpha\right)-\left(r^{\prime}-i^{\prime}\right)$ colors, which can be used to select AV0-5 Main Sequence star candidates (age 20-200 Myr). By combining a sample of 23050 IPHAS-selected A-type stars with 2MASS, GLIMPSE and MIPSGAL photometry we searched for mid-infrared excesses attributable to dusty circumstellar disks. Positional cross-correlation yielded a sample of 2692 A-type stars, of which $0.6 \%$ were found to have $8-\mu \mathrm{m}$ excesses above the expected photospheric values. The low fraction of main sequence stars with mid-IR excesses found in this work indicates that dust disks in the terrestrial planet zone of Main Sequence intermediate mass stars are rare. Dissipation mechanisms such as photo-evaporation, grain growth, collisional grinding or planet formation could possibly explain the depletion of dust detected in the inner regions of these disks.
\end{abstract}

Keywords. Stars: circumstellar matter; Planetary systems: proto-planetary disks

From the observed colors in the $\left(\mathrm{r}^{\prime}-\mathrm{H} \alpha\right)-\left(r^{\prime}-i^{\prime}\right)$ plane, we identify 23050 early A-type main sequence (MS) star candidates in the Isaac Newton Photometric H-Alpha Survey (IPHAS, Drew et al. 2005). Positional cross-correlation with the 2MASS and GLIMPSE catalogs led to a sample of 2692 candidate A-type stars with fully sampled 0.6 to $8 \mu \mathrm{m}$ SEDs. Optical classification spectra obtained of 10 of the systems confirmed that all but one were main sequence A-type stars, the exception being an A5Ia super-giant. We searched for stars with 8 - $\mu$ m excesses by looking for unusually large $(K-8)$ colors. 17 stars $(0.6 \%)$ were found to have $8-\mu \mathrm{m}$ excesses above the expected photospheric value (Hales et al. 2009). Free-free emission from ionized gas can cause mid-IR excesses around B stars. However, these stars will also show prominent $\mathrm{H} \alpha$ emission (Uzpen et al. 2008), while our IPHAS-based color selection method ensures that stars in our sample have undetectable levels of $\mathrm{H} \alpha$ emission. Therefore the mid-IR excesses in our sample are most likely to originate in a disk of warm dust located close to the central stars $(\mathrm{r}<$ $1 \mathrm{AU}) .10$ out of the 17 excess stars had been covered by Spitzer MIPSGAL survey fields, of which 5 had detectable excesses at $24 \mu \mathrm{m}$. For sources with excesses detected in at least two mid-IR wavelength bands, blackbody fits to the excess SEDs yielded temperatures ranging from 270 to $650 \mathrm{~K}$, and bolometric luminosity ratios comparable to those of warm debris-disk systems, similar to $\beta$ Pictoris.

\section{References}

Drew, J. E. et al. 2005, MNRAS, 362, 753

Hales, A. S. et al., 2009, ApJ, 695, 75

Uzpen, B., Kobulnicky, H. A., Semler, D. R., Bensby, T., \& Thom, C. 2008, ApJ, 685, 1157 